\title{
High Performance and Energy Efficient Traffic Light Controller Design Using FPGA
}

Sujeet Pandey ${ }^{1}$, Vivek Kumar Shrivastav ${ }^{2}$, Rashmi Sharma ${ }^{3}$, D M

\author{
Akbar Hussain ${ }^{4}$ \\ ${ }^{1,3}$ Center of Energy Excellence, Gyancity Research Lab, Motihari, India \\ ${ }^{2}$ Dept of Aeronautical Engineering, Indian Institute of Kharagpur, Kharagapur, India \\ ${ }^{4}$ Aalborg University, Esbjerg, Denmark \\ sujeet@gyancity.com, aerovivek91@gmail.com, rashmisharma1505@gmail.com,
}

akh@et.aau.dk

\begin{abstract}
In this work, Verilog is used as hardware description language for implementation of traffic light controller. It shows Red, Green and Yellow color at a predefined interval. Technology scaling is used as energy efficient technique. We have used 90nm, 65nm, 40nm and $28 \mathrm{~nm}$ technology based FPGA and then we have analyzed power consumption for traffic light controller on different FPGA. Leakage power is in range of 97.5-99\% of total power consumption by traffic light controller on Virtex-7 FPGA. Signal power, clock power and IOs power are almost negligible. Power dissipation is measured on XPOWER simulator.
\end{abstract}

Keywords: Traffic Light Controller, FPGA, Verilog, Energy Efficient Design

\section{Introduction}

Traffic light controllers are used to control the movement of the vehicles. In the FPGA implementation of Traffic Light Controller (as shown in Fig.1-3), we are using Virtex-4, Virtex-5, Virtex-6 and Virtex-7 FPGA. Virtex-4 is 90nm technology based FPGA. Virtex5 is $65 \mathrm{~nm}$ technology based FPGA. Virtex-6 is 40nm technology based FPGA. Virtex-7 is $28 \mathrm{~nm}$ technology based FPGA. LVCMOS18 is the default IO standards. 12 is drive strength as shown in Table 1 . There are two inputs and 12 outputs in design under consideration. We have generated RTL schematic, technology schematic. Section 1 is introduction. Section 2 is related works on FPGA. Section 3 is results of Xpower simulator that measure power in terms of signal power, clock power, logic power, IO power and leakage power. Total power is sum of signal power, clock power, logic power, IO power and leakage power. Y denotes Yellow, R denotes Red and G denotes Green color of traffic light controller. 


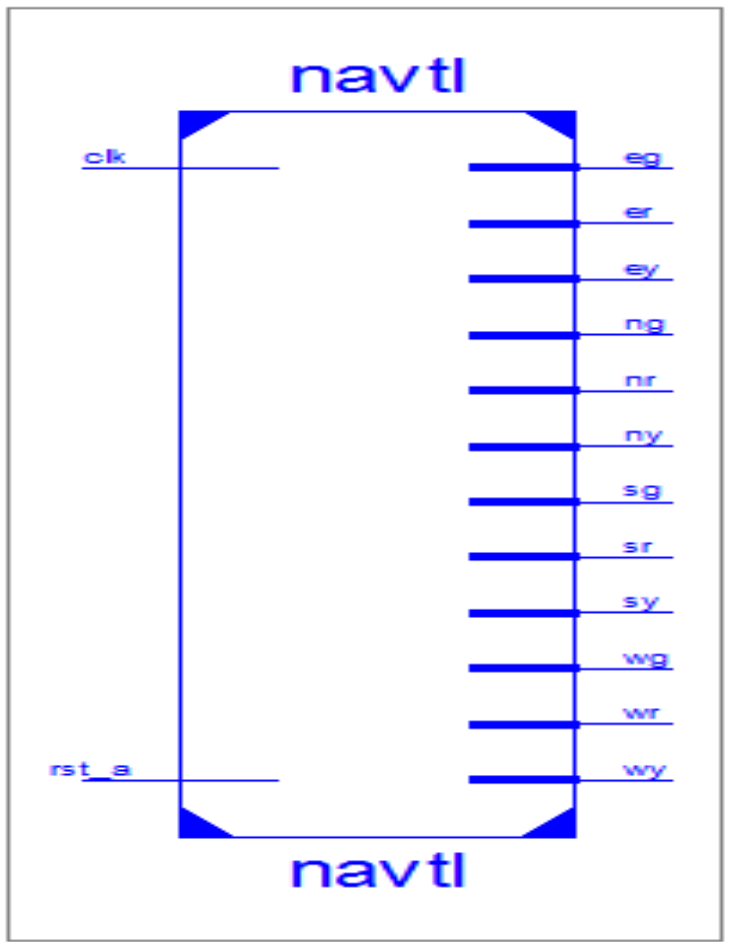

Figure 1: RTL Schematics of Traffic Light Controller

Top Level of RTL schematic is shown in Fig.1. It shows basic input required for traffic light controller and basic output required for our design under test. Clock and reset are two inputs. Whereas, there are twelve output as shown in Fig. 1 and Table 1.

Table 1: IOB properties of Traffic Light Controller

\begin{tabular}{|l|l|l|l|l|l|}
\hline $\begin{array}{l}\text { IOB } \\
\text { Name }\end{array}$ & Type & Direction & IO Standard & $\begin{array}{l}\text { Drive } \\
\text { Strength }\end{array}$ & $\begin{array}{l}\text { Slew } \\
\text { Rate }\end{array}$ \\
\hline clk & IOB & INPUT & LVCMOS18 & \multicolumn{2}{|l|}{} \\
\hline eg & IOB & OUTPUT & LVCMOS18 & 12 & SLOW \\
\hline er & IOB & OUTPUT & LVCMOS18 & 12 & SLOW \\
\hline ey & IOB & OUTPUT & LVCMOS18 & 12 & SLOW \\
\hline ng & IOB & OUTPUT & LVCMOS18 & 12 & SLOW \\
\hline nr & IOB & OUTPUT & LVCMOS18 & 12 & SLOW \\
\hline ny & IOB & OUTPUT & LVCMOS18 & 12 & SLOW \\
\hline rst_a & IOB & INPUT & LVCMOS18 & \multicolumn{1}{|l|}{} \\
\hline sg & IOB & OUTPUT & LVCMOS18 & 12 & SLOW \\
\hline sr & IOB & OUTPUT & LVCMOS18 & 12 & SLOW \\
\hline sy & IOB & OUTPUT & LVCMOS18 & 12 & SLOW \\
\hline wg & IOB & OUTPUT & LVCMOS18 & 12 & SLOW \\
\hline wr & IOB & OUTPUT & LVCMOS18 & 12 & SLOW \\
\hline wy & IOB & OUTPUT & LVCMOS18 & 12 & SLOW \\
\hline
\end{tabular}


Internal Architecture of RTL Schematic of traffic light controller is shown in Fig. 2. This architecture shows different multiplexer, gates and other basic primitive circuits used in RTL schematic. Technology schematic of our design is shown in Fig. 3.

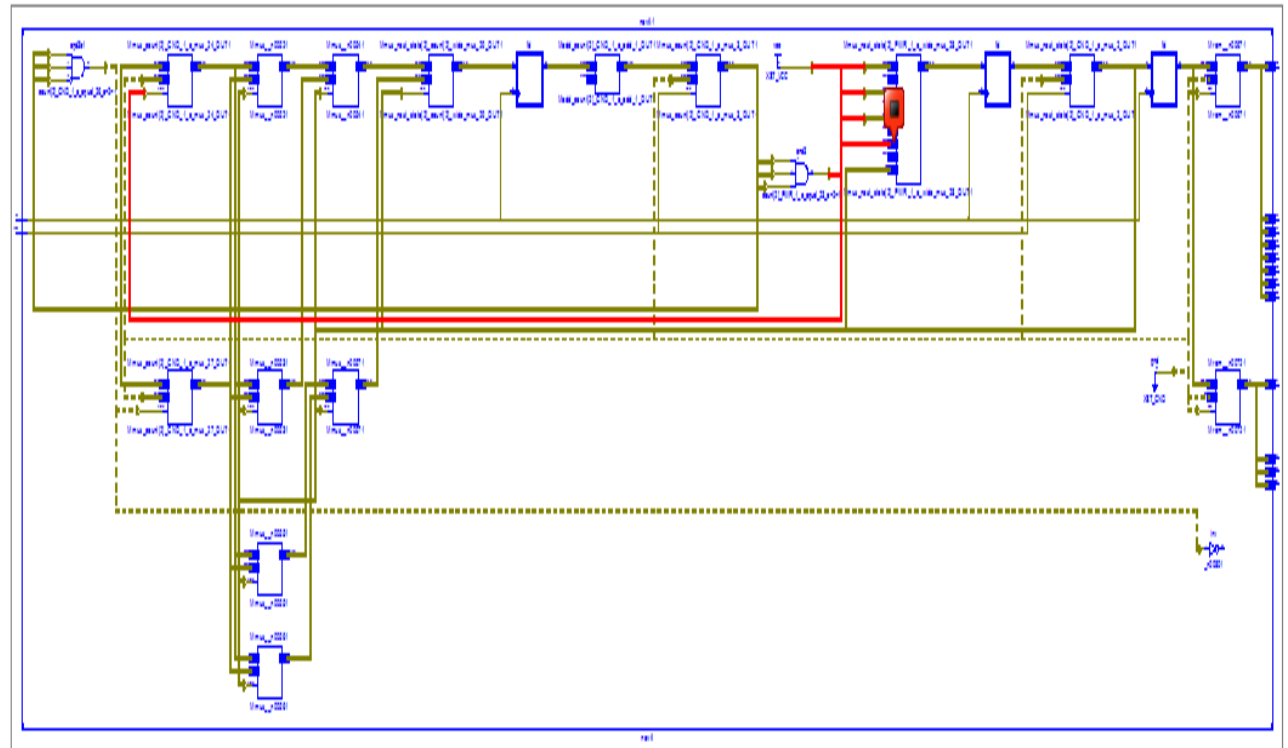

Figure 2: Internal Architecture of RTL Schematic

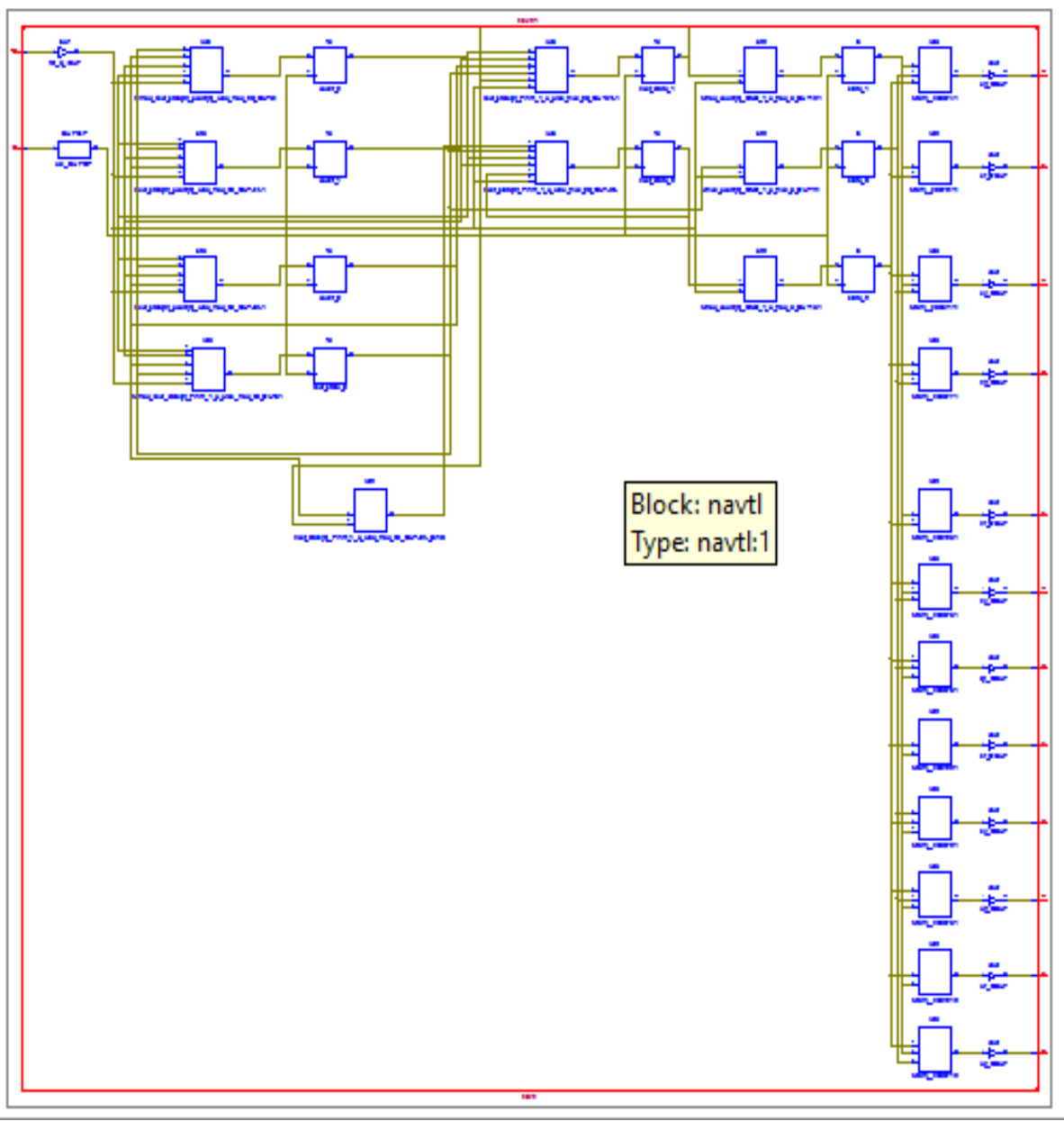

Figure 3: Technology Schematic of Traffic Light Controller 


\section{Related Works}

A Novel Noise Free Transmission Technique is used for design of 100Gb/s Future Generation Optical Communication System on FPGA [1]. Different I/O standard and technology like thermal aware techniques and energy efficient techniques are used in Vedic multiplier design for green wireless communication on FPGA [2]. Mobile DDR IO standard is used in high performance energy efficient portable ALU design on FPGA [3]. HSTL IO Standard is used in energy efficient multiplier design on 28nm FPGA [4]. Capacitance scaling, Thermal scaling, HSTL IO standards and SSTL IO standards are used in energy efficient thermal aware image ALU design on FPGA [5]. Low power consumption techniques on FPGA is discussed for wireless devices [6]. We evaluate performance of FIR Filter and also implement on different FPGA and SOC [7]. IoTs enable Object Tracking on FPGA is discussed in [8].

\section{Results}

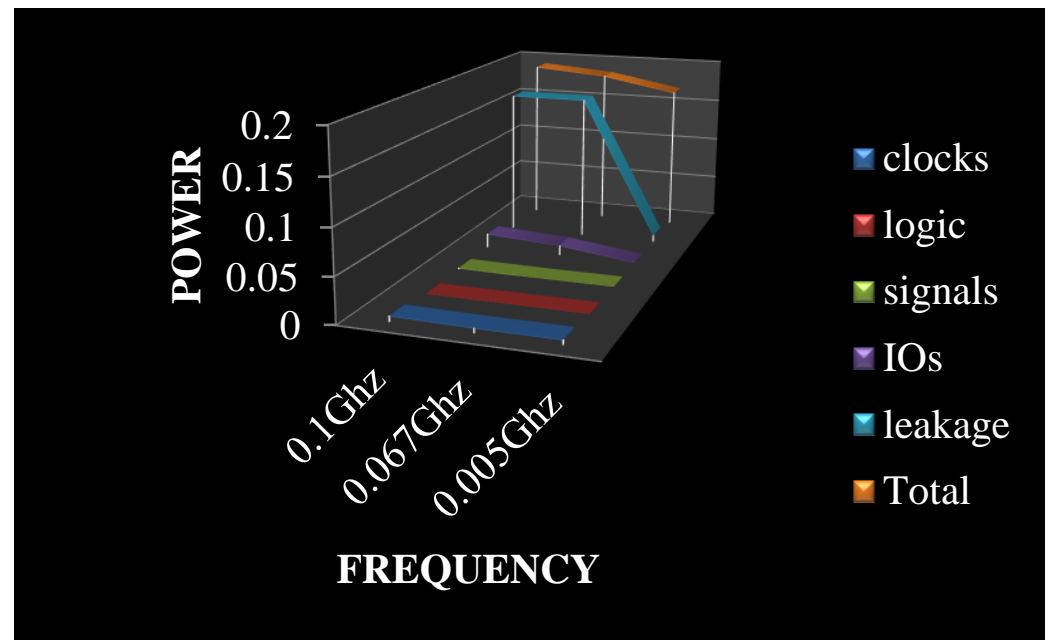

Figure 4: Power Dissipation of Traffic Light Controller with Virtex-4 FPGA

Clock power is directly proportional to frequency. Clock power increase with increase in frequency and decrease with decrease in frequency. Logic power is significant for $0.2 \mathrm{GHz}$ and it becomes negligible for lower frequency as shown in Fig.4 and Table 2.

Table 2: Power Dissipation of Traffic Light Controller with Virtex-4 FPGA

\begin{tabular}{|l|l|l|l|l|l|l|}
\hline $\begin{array}{l}\text { Power } \rightarrow \\
\text { Frequency } \downarrow\end{array}$ & Clock & Logic & Signals & IOs & Leakage & Total \\
\hline $0.2 \mathrm{GHz}$ & 0.007 & 0.001 & 0.001 & 0.032 & 0.167 & 0.208 \\
\hline $0.1 \mathrm{GHz}$ & 0.006 & 0.000 & 0.001 & 0.016 & 0.167 & 0.190 \\
\hline $0.067 \mathrm{GHz}$ & 0.005 & 0.000 & 0.000 & 0.011 & 0.167 & 0.183 \\
\hline $0.005 \mathrm{GHz}$ & 0.005 & 0.000 & 0.000 & 0.000 & 0.008 & 0.167 \\
\hline
\end{tabular}




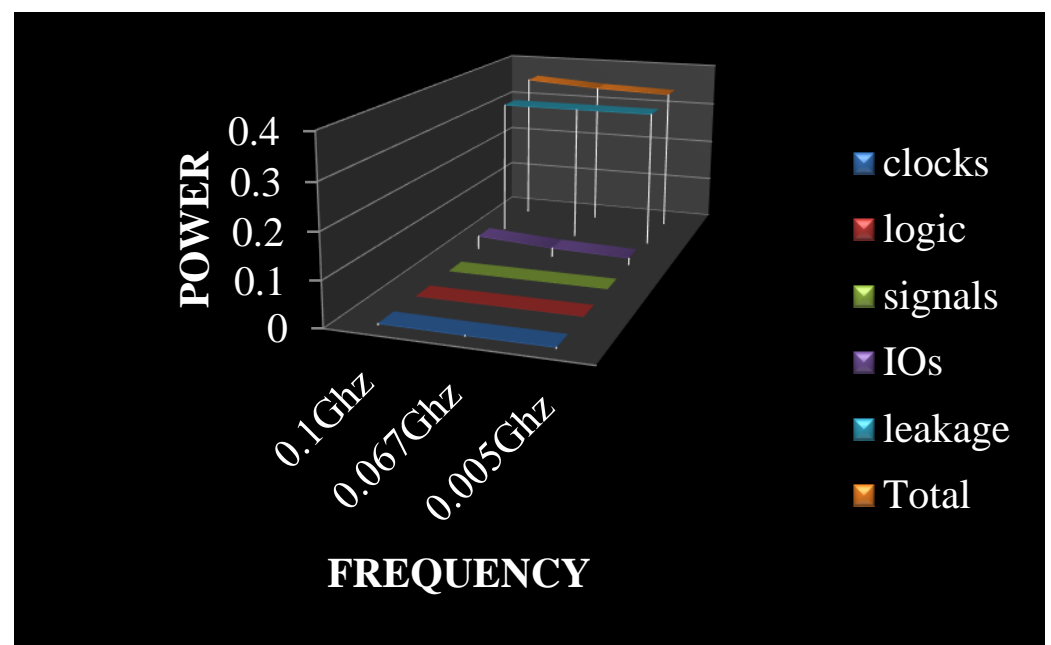

Figure 5: Power Dissipation of Traffic Light Controller with Virtex-5 FPGA

On Virtex-5, clock power is lower that clock power dissipation on Virtex-4 FPGA. Leakage power, IO Power and Total power on Virtex-5 is higher than leakage power dissipation on Virtex-4 FPGA. IOs power dissipation by traffic light controller is lower than leakage power as shown in Table 3 and Fig.5.

Table 3: Power Dissipation of Traffic Light Controller with Virtex-5 FPGA

\begin{tabular}{|l|l|l|l|l|l|l|}
\hline $\begin{array}{l}\text { Power } \rightarrow \\
\text { Frequency } \\
\end{array}$ & Clock & Logic & Signals & IOs & Leakage & Total \\
\hline $0.2 \mathrm{Ghz}$ & 0.005 & 0.001 & 0.001 & 0.064 & 0.321 & 0.392 \\
\hline $0.1 \mathrm{Ghz}$ & 0.003 & 0.000 & 0.000 & 0.032 & 0.321 & 0.357 \\
\hline $0.067 \mathrm{Ghz}$ & 0.002 & 0.000 & 0.000 & 0.021 & 0.321 & 0.345 \\
\hline $0.005 \mathrm{Ghz}$ & 0.002 & 0.000 & 0.000 & 0.016 & 0.321 & 0.339 \\
\hline
\end{tabular}

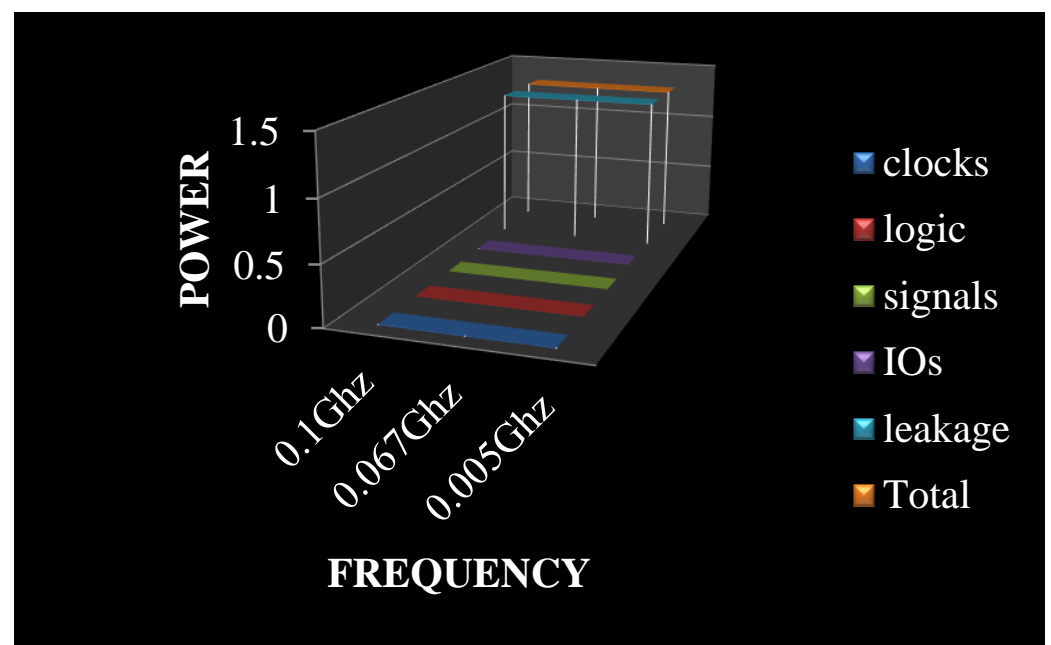

Figure 6: Power Dissipation of Traffic Light Controller with Virtex-6 FPGA

Leakage power is a maximum contributor in total power dissipation by traffic light controller on Virtex-6 FPGA. Clock power is the second most contributor in total power 
dissipation. Logic, Signals and IOs power dissipation by traffic light controller is negligible as shown in Fig. 6 and Table 4.

Table 4: Power Dissipation of Traffic Light Controller with Virtex-6 FPGA

\begin{tabular}{|l|l|l|l|l|l|l|}
\hline $\begin{array}{l}\text { Power } \rightarrow \\
\text { Frequency } \downarrow\end{array}$ & Clock & Logic & Signals & IOs & Leakage & Total \\
\hline $0.2 \mathrm{Ghz}$ & 0.006 & 0.000 & 0.000 & 0.001 & 1.293 & 1.301 \\
\hline $0.1 \mathrm{Ghz}$ & 0.003 & 0.000 & 0.000 & 0.001 & 1.293 & 1.297 \\
\hline $0.067 \mathrm{Ghz}$ & 0.002 & 0.000 & 0.000 & 0.000 & 1.293 & 1.295 \\
\hline $0.005 \mathrm{Ghz}$ & 0.002 & 0.000 & 0.000 & 0.000 & 1.293 & 1.295 \\
\hline
\end{tabular}

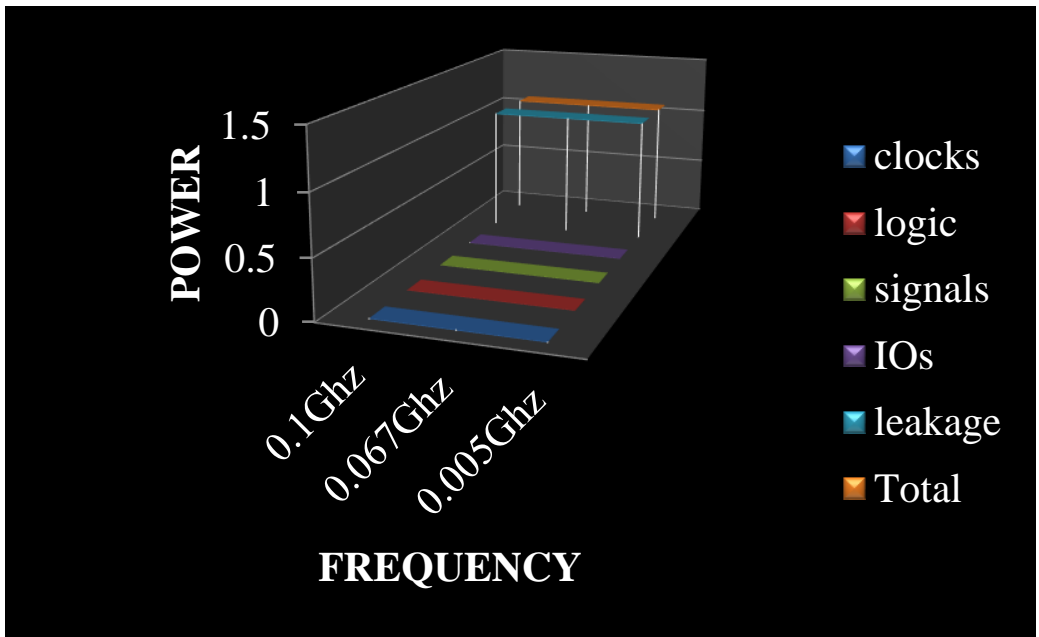

Figure 7: Power Dissipation of Traffic Light Controller with Virtex-6 Low Power FPGA

Virtex-6 Low Power FPGA has low power dissipation than the simple Virtex-6 FPGA especially in leakage power dissipation. Clock, logic, signals and IOs power dissipation are almost equal as shown in Fig.7 and Table 5.

Table 5: Power Dissipation of Traffic Light Controller with Virtex-6 Low Power FPGA

\begin{tabular}{|l|l|l|l|l|l|l|}
\hline $\begin{array}{l}\text { Power } \rightarrow \\
\text { Frequency } \downarrow\end{array}$ & Clock & Logic & Signals & IOs & Leakage & Total \\
\hline $0.2 \mathrm{Ghz}$ & 0.006 & 0.000 & 0.000 & 0.001 & 1.065 & 1.072 \\
\hline $0.1 \mathrm{Ghz}$ & 0.003 & 0.000 & 0.000 & 0.001 & 1.065 & 1.069 \\
\hline $0.067 \mathrm{Ghz}$ & 0.002 & 0.000 & 0.000 & 0.000 & 1.065 & 1.068 \\
\hline $0.005 \mathrm{Ghz}$ & 0.001 & 0.000 & 0.000 & 0.000 & 1.065 & 1.067 \\
\hline
\end{tabular}




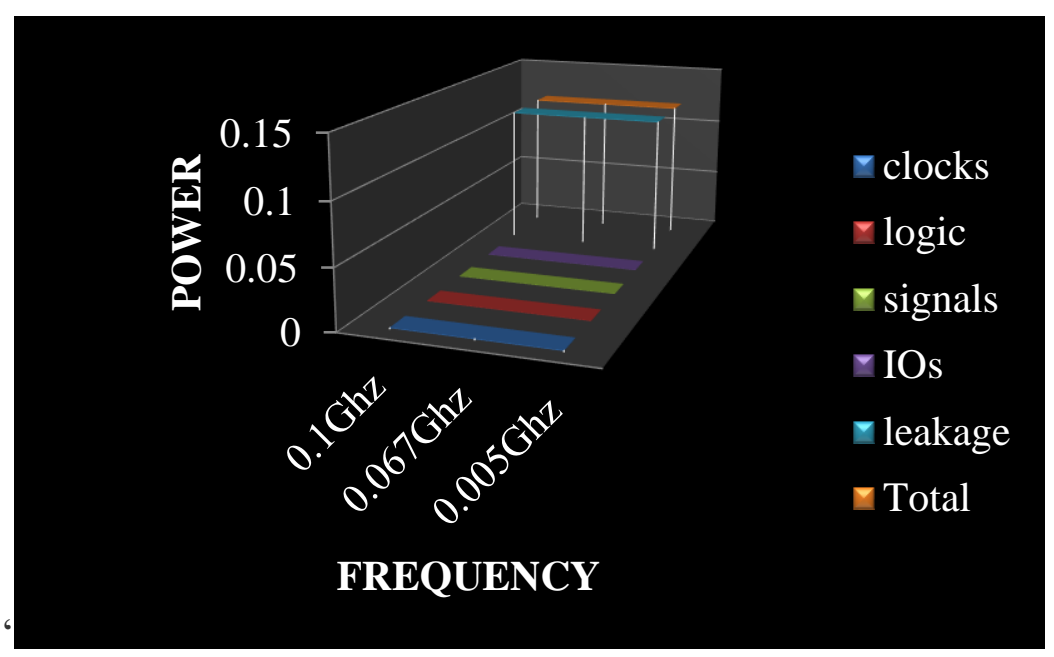

Figure 8: Power Dissipation of Traffic Light Controller with Virtex-7 Low Voltage FPGA

Leakage power is in range of $97.5-99 \%$ of total power consumption by traffic light controller on Virtex-7 FPGA as shown in Fig.8 and Table 6.

Table 6: Power Dissipation of Traffic Light Controller with Virtex-7 Low Voltage FPGA

\begin{tabular}{|l|l|l|l|l|l|l|}
\hline $\begin{array}{l}\text { Power } \rightarrow \\
\text { Frequency } \downarrow\end{array}$ & Clock & Logic & Signals & IOs & Leakage & Total \\
\hline $0.2 \mathrm{Ghz}$ & 0.002 & 0.000 & 0.000 & 0.001 & 0.117 & 0.120 \\
\hline $0.1 \mathrm{Ghz}$ & 0.001 & 0.000 & 0.000 & 0.000 & 0.117 & 0.118 \\
\hline $0.067 \mathrm{Ghz}$ & 0.001 & 0.000 & 0.000 & 0.000 & 0.117 & 0.118 \\
\hline $0.005 \mathrm{Ghz}$ & 0.001 & 0.000 & 0.000 & 0.000 & 0.117 & 0.118 \\
\hline
\end{tabular}

\section{Conclusion and Future Scope}

In this work we are using 90nm, 65nm, 40nm and 28nm technology based FPGA. In future, we shall use ultrascale FPGA for implementation of traffic light controller.

\section{References}

[1]. Bhagwan Das, MFL Abdullah, Bishwajeet Pandey, DMA Hussain and BS Chowdhry, "A Novel Noise Free Transmission Technique for Designing 100Gb/s Future Generation Optical Communication System", International Journal of Future Generation and Communication Network, (pp. 33-40).

[2]. Goswami, K., Pandey, B., Kumar, T. et al., "Different I/O Standard and Technology Based Thermal Aware Energy Efficient Vedic Multiplier Design for Green Wireless Communication on FPGA", Wireless Pers Commun, September 2017, Volume 96, Issue 2, pp 31393158. https://doi.org/10.1007/s11277-017-4345-6

[3]. Tanesh Kumar, Bishwajeet Pandey, Teerath Das, and Bhavani Shankar Chowdhry, "Mobile DDR IO Standard Based High Performance Energy Efficient Portable ALU Design on FPGA", Wireless Personal Communications, An International Journal, Volume 76, Issue 3 (2014), Page 569-578

[4]. Shivani Madhok, Bishwajeet Pandey and Amanpreet Kaur, Mohamed Hashim Minver and D M Akbar Hussain, "HSTL IO Standard Based Energy Efficient Multiplier Design using Nikhilam Navatashcaramam Dashatah on 28nm FPGA", International Journal of Control and Automation Vol.8, No.8 (2015), pp.35-44

[5]. Tanesh Kumar, Bishwajeet Pandey, S. H. A. Mussavi, and Noor Zaman. "CTHS based energy efficient thermal aware image ALU design on FPGA." Wireless Personal Communications, Vol. 85, No. 3, December 2015, Page: 671-696. 
[6]. Gaurav Verma, Manish Kumar, Vijay Khare, Bishwajeet Pandey, "Analysis of Low Power Consumption Techniques on FPGA for Wireless Devices", Wireless Personal Communications, (SCIE), July 2017 ,
Volume
95 ,
Issue
2 ,
pp
$353-$

364. http://link.springer.com/article/10.1007/s11277-016-3896-2

[7]. Bishwajeet Pandey, Bhagwan Das, Amanpreet Kaur, Tanesh Kumar, Abdul Moid Khan, D M Akbar Hussain, Geetam S Tomar, "Performance Evaluation of FIR Filter After Implementation on Different FPGA and SOC and Its Utilization in Communication and Network", Wireless Personal Communications, (SCIE), July 2017, Volume 95, Issue 2, pp 375389. http://link.springer.com/article/10.1007/s11277-016-3898-0

[8]. Sayed Hyder Abbas Musavi, B. S. Chowdhry, Tanesh Kumar, Bishwajeet Pandey, Wanod Kumar, "IoTs Enable Active Contour Modeling Based Energy Efficient and Thermal Aware Object Tracking on FPGA", Wireless Personal Communications, Vol.85, No.2, pp.529-543, 20 May 2015, (SCIE) http://link.springer.com/article/10.1007/s11277-015-2753-Z 